\title{
Ishikawa-hybrid proximal point algorithm for NSVI system
}

\author{
Hong Gang Li* and Min Qiu
}

"Correspondence: lihg12@126.com College of Mathematics and Physics, Chongqing University of Posts and Telecommunications, Chongqing, 400065, China

\begin{abstract}
A nonlinear set-valued inclusions system framework for an Ishikawa-hybrid proximal point algorithm is developed and studied using the notion of an $(A, \eta)$-accretive mapping. Convergence analysis for the algorithm of solving the nonlinear set-valued inclusions system and existence analysis of solution for the system are explored along with some results on the resolvent operator corresponding to the $(A, \eta)$-accretive mapping in a Banach space. The result that the sequence generated by the algorithm converges linearly to a solution of the system with the convergence rate $\|\boldsymbol{\Psi}\|$ is proved.
\end{abstract}

MSC: 49J40;47H06

Keywords: nonlinear set-valued inclusions system; $(A, \eta)$-accretive mapping; resolvent operator; Ishikawa-hybrid proximal point algorithm; convergence rate

\section{Introduction}

The nonlinear set-valued inclusions system, which was introduced and studied by Hassouni and Moudafi [1], is a useful and important extension of the variational inequality and variational inclusions system. In recent years, various variational inclusions systems and nonlinear set-valued inclusions systems have been intensively studied. For example, Kassay and Kolumbán [2], Chen, Deng and Tan [3], Yan, Fang and Huang [4], Fang, Huang and Thompson [5], Jin [6], Verma [7], Li, Xu and Jin [8], Kang, Cho and Liu [9] et al. introduced and studied various set-valued variational inclusions systems. For the past few years, many existence results and iterative algorithms for various variational inclusions systems have been studied. For details, please see [1-28] and the references therein.

Example 1.1 In 2001, Chen, Deng and Tan [3] have studied the problem associated with the following system of variational inequalities, which is finding $(x, y) \in H \times H(H$, Hilbert space) such that

$$
\left\{\begin{array}{l}
\langle\rho T(y)+x-y, w-x\rangle \geq \rho \varphi_{1}(x)-\rho \varphi_{1}(w), \\
\langle t S(x)+y-x, w-y\rangle \geq t \varphi_{2}(y)-\rho \varphi_{2}(w) \quad(\forall w \in H),
\end{array}\right.
$$

where $\varphi_{i}: H \rightarrow R \cup \infty$ is a proper, convex, lower semicontinuous functional and $\partial \varphi_{i}(\cdot)$ denotes the subdifferential operator of $\varphi_{i}(i=1,2)$.

@ 2012 Li and Qiu; licensee Springer. This is an Open Access article distributed under the terms of the Creative Commons Attribution License (http://creativecommons.org/licenses/by/2.0), which permits unrestricted use, distribution, and reproduction in any medium, provided the original work is properly cited. 
Example 1.2 Let $X$ be a real $q$-uniformly smooth Banach space, and $S, T, M_{1}, M_{2}: X \rightarrow X$ be four single-valued mappings. Find $x, y \in X$ such that

$$
\left\{\begin{array}{l}
0 \in x-y+\rho T(y)+\rho M_{1}(x), \\
0 \in y-x+t S(x)+t M_{2}(y),
\end{array}\right.
$$

which is studied by Jin in [6].

Inspired and motivated by Examples 1.1-1.2 and recent research work in this field (see $[7,8])$, in this paper, we will introduce and discuss the problem associated with the following class of new nonlinear set-valued inclusions systems (NSVI Systems), which is finding $(x, y) \in X \times X$ for any $f, g: X \rightarrow X$ such that $z \in S(x), w \in T(y)$, and

$$
\left\{\begin{array}{l}
f(x) \in F(z, y)+M(y), \\
g(y) \in G(w, x)+N(x),
\end{array}\right.
$$

where $X$ is a real $q$-uniformly smooth Banach space, $A, B: X \rightarrow X, \eta_{1}, \eta_{2}: X \times X \rightarrow X$, and $F, G: X \times X \rightarrow X$ are single-valued mappings; $M: X \rightarrow 2^{X}$ is a set-valued $\left(A, \eta_{1}\right)$-accretive mapping and $N: X \rightarrow 2^{X}$ is a set-valued $\left(B, \eta_{2}\right)$-accretive mapping, and $S, T: X \rightarrow C B(X)$ are two set-valued mappings.

If $f(x)=-x, g(y)=-y, F(w, y)=\rho T(y)-y, G(y, x)=t S(x)-x, N=t M_{2}$ and $M=\rho M_{1}(\cdot)$, then the problem (3) reduces to Example 1.1. If $M_{1}=M_{2}=\partial \varphi, \varphi_{i}: H \rightarrow R \cup \infty$ is a proper, convex, lower semicontinuous functional and $\partial \varphi_{i}(\cdot)$ denotes the subdifferential operator of $\varphi_{i}(X=H$, Hilbert space, and $i=1,2)$, then the problem (3) changes to Example 1.2 .

If $X$ is a real $q$-uniformly smooth Banach space, and $G(\cdot, \cdot)=N(y, g(x)), f(u)=u$ and $S(u)=Q(u)(u \in X)$, then the problem (3) reduces to the problem associated with the following variational inclusions:

For any $u \in X$, find $x \in X$ and $y=Q(x)$ such that

$$
u \in N(y, g(x))+M(y)
$$

which is developed by Li in 2010 [8].

The main purpose of this paper is to introduce and study a generalized nonlinear setvalued inclusions system framework for an Ishikawa-hybrid proximal point algorithm using the notion of $(A, \eta)$-accretive due to Lan-Cho-Verma [10] in a Banach space, to analyse convergence for the algorithm of solving the system and existence of a solution for the system and to prove the result that sequence $\left\{\left(x^{n}, y^{n}\right)\right\}_{n=0}^{\infty}$ generated by the algorithm converges linearly to a solution of the nonlinear set-valued inclusions system with the convergence rate $\|\Psi\|$.

\section{Preliminaries}

Let $X$ be a real $q$-uniformly smooth Banach space with a dual space $X^{*},\langle\cdot, \cdot\rangle$ be the dual pair between $X$ and $X^{*}, 2^{X}$ denote the family of all the nonempty subsets of $X$, and $C B(X)$ denote the family of all nonempty closed bounded subsets of $X$. The generalized duality 
mapping $J_{q}: X \rightarrow 2^{X^{*}}$ is defined by

$$
J_{q}(x)=\left\{f^{*} \in X^{*}:\left\langle x, f^{*}\right\rangle=\|x\|^{q},\left\|f^{*}\right\|=\|x\|^{q-1}\right\}, \quad \forall x \in X,
$$

where $q>1$ is a constant. Let us recall the following results and concepts.

Definition 2.1 A single-valued mapping $\eta: X \times X \rightarrow X$ is said to be $\tau$-Lipschitz continuous if there exists a constant $\tau>0$ such that

$$
\|\eta(x, y)\| \leq \tau\|x-y\|, \quad \forall x, y \in X .
$$

Definition 2.2 A single-valued mapping $A: X \rightarrow X$ is said to be

(i) accretive if

$$
\left\langle A\left(x_{1}\right)-A\left(x_{2}\right), J_{q}\left(x_{1}-x_{2}\right)\right\rangle \geq 0, \quad \forall x_{1}, x_{2} \in X
$$

(ii) strictly accretive if $A$ is accretive and $\left\langle A\left(x_{1}\right)-A\left(x_{2}\right), J_{q}\left(x_{1}-x_{2}\right)\right\rangle=0$ if and only if $x_{1}=x_{2}, \forall x_{1}, x_{2} \in X$

(iii) $r$-strongly $\eta$-accretive if there exists a constant $r>0$ such that

$$
\left\langle A\left(x_{1}\right)-A\left(x_{2}\right), J_{q}\left(\eta\left(x_{1}, x_{2}\right)\right)\right\rangle \geq r\left\|x_{1}-x_{2}\right\|^{q}, \quad \forall x_{1}, x_{2} \in X
$$

(iv) $\gamma$-Lipschitz continuous if there exists a constant $\gamma>0$ such that

$$
\left\|A\left(x_{1}\right)-A\left(x_{2}\right)\right\| \leq \gamma\left\|x_{1}-x_{2}\right\|, \quad \forall x_{1}, x_{2} \in X
$$

(v) Let $f: X \rightarrow X$ be a single-valued mapping. $A$ is said to be $(\sigma, \varphi)$-relaxed cocoercive with respect to $f$ if for any $x_{1}, x_{2} \in X$, there exist two constants $\sigma, \varphi>0$ such that

$$
\left\langle A\left(x_{1}\right)-A\left(x_{2}\right), J_{q}\left(f\left(x_{1}\right)-f\left(x_{2}\right)\right)\right\rangle \geq-\sigma\left\|A\left(x_{1}\right)-A\left(x_{2}\right)\right\|^{q}+\varphi\left\|x_{1}-x_{2}\right\|^{q} .
$$

Definition 2.3 A set-valued mapping $S: X \rightarrow C B(X)$ is said to be

(i) $D$-Lipschitz continuous if there exists a constant $\alpha>0$ such that

$$
D(S(x), S(y)) \leq \alpha\|x-y\|, \quad \forall x, y \in X
$$

where $D(\cdot, \cdot)$ is the Hausdorff metric on $C B(X)$.

(ii) $\beta$-strongly $\eta$-accretive if there exists a constant $\beta>0$ such that

$$
\left\langle u_{1}-u_{2}, J_{q}(\eta(x, y))\right| \geq \beta\|x-y\|^{q}, \quad \forall x, y \in X, u_{1} \in S(x), u_{2} \in S(y) .
$$

Definition 2.4 Let $A: X \rightarrow X$ and $\eta: X \times X \rightarrow X$ be single-valued mappings. A set-valued mapping $M: X \rightarrow 2^{X}$ is said to be

(i) accretive if

$$
\left\langle u_{1}-u_{2}, J_{q}(x, y)\right| \geq 0, \quad \forall x, y \in X, u_{1} \in M(x), u_{2} \in M(y)
$$


(ii) $\eta$-accretive if

$$
\left\langle u_{1}-u_{2}, J_{q}(\eta(x, y))\right\rangle \geq 0, \quad \forall x, y \in X, u_{1} \in M(x), u_{2} \in M(y)
$$

(iii) $m$-relaxed $\eta$-accretive, if there exists a constant $m>0$ such that

$$
\left\langle u_{1}-u_{2}, J_{q}(\eta(x, y))\right\rangle \geq-m\|x-y\|^{q}, \quad \forall x, y \in X, u_{1} \in M(x), u_{2} \in M(y)
$$

(iv) $A$-accretive if $M$ is accretive and $(A+\rho M)(X)=X$ for all $\rho>0$;

(v) $(A, \eta)$-accretive if $M$ is $m$-relaxed $\eta$-accretive and $(A+\rho M)(X)=X$ for every $\rho>0$.

Based on [10], we can define the resolvent operator $R_{\rho, M}^{A, \eta}$ as follows.

Lemma 2.5 ([10]) Let $\eta: X \times X \rightarrow X$ be a $\tau$-Lipschitz continuous mapping, $A: X \rightarrow X$ be an $r$-strongly $\eta$-accretive mapping, and $M: X \rightarrow 2^{X}$ be a set-valued $(A, \eta)$-accretive mapping. Then the generalized resolvent operator $R_{\rho, M}^{A, \eta}: X \rightarrow X$ is $\tau^{q-1} /(r-m \rho)$-Lipschitz continuous; that is,

$$
\left\|R_{\rho, M}^{A, \eta}(x)-R_{\rho, M}^{A, \eta}(y)\right\| \leq \frac{\tau^{q-1}}{r-m \rho}\|x-y\| \quad \text { for all } x, y \in X,
$$

where $\rho \in(0, r / m), q>1$.

Remark 2.6 The $(A, \eta)$-accretive mappings are more general than $(H, \eta)$-monotone mappings, $A$-monotone operators and $\eta$-subdifferential operators in a Banach space or a Hilbert space, and the resolvent operators associated with $(A, \eta)$-accretive mappings include as special cases the corresponding resolvent operators associated with them, respectively $[3-6,9,25]$.

In the study of characteristic inequalities in $q$-uniformly smooth Banach spaces $X, \mathrm{Xu}$ [14] proved the following result.

Lemma 2.7 ([14]) Let $X$ be a real uniformly smooth Banach space. Then $X$ is q-uniformly smooth if and only if there exists a constant $c_{q}>0$ such that for all $x, y \in X$,

$$
\|x+y\|^{q} \leq\|x\|^{q}+q\left\langle y, J_{q}(x)\right\rangle+c_{q}\|y\|^{q} .
$$

Lemma 2.8 ([8]) Let $a, b, c>0$ be real, for any real $q \geq 1$, if $a^{q} \leq b^{q}+c^{q}$, then

$$
a \leq b+c
$$

\section{Existence theorem of solutions}

Let us study the existence theorem of solutions for the inclusions system (3).

Theorem 3.1 Let $X$ be a Banach space, $f, g: X \rightarrow X$ be two single-valued mappings, $F: X \times X \rightarrow X$ be $a\left(\mu_{1}, v_{1}\right)$-Lipschitz continuous mapping and $G: X \times X \rightarrow X$ be $a\left(\mu_{2}, v_{2}\right)$ Lipschitz continuous mapping, $\eta_{i}: X \times X \rightarrow X$ be a $\tau_{i}$-Lipschitz continuous mapping $(i=1,2), A: X \rightarrow X$ be an $r_{1}$-strongly $\eta_{1}$-accretive mapping, $B: X \rightarrow X$ be an $r_{2}$-strongly $\eta_{2}$-accretive mapping, $M: X \rightarrow 2^{X}$ be a set-valued $\left(A, \eta_{1}\right)$-accretive mapping and $N: X \rightarrow$ $2^{X}$ be a set-valued $\left(B, \eta_{2}\right)$-accretive mapping. Then the following statements are mutually 
equivalent:

(i) An element $(x, y)$ is a solution of the problem (3);

(ii) For $(x, y) \in X \times X, z \in S(x)$ and $w \in T(y)$, the following relations hold:

$$
\left\{\begin{array}{l}
x=R_{\rho_{1}, M}^{A, \eta_{1}}\left(A(x)+\rho_{1} f(x)-\rho_{1} F(z, y)\right) \\
y=R_{\rho_{2}, N}^{B, \eta_{2}}\left(B(y)+\rho_{2} g(y)-\rho_{2} G(w, x)\right)
\end{array}\right.
$$

where $\rho_{i}>0$ is a constant $(i=1,2)$;

(iii) For $(x, y) \in X \times X, z \in S(x), w \in T(y)$, and any $1>\lambda>0$, the following relations hold:

$$
\left\{\begin{array}{l}
x=(1-\lambda) x+\lambda R_{\rho_{1}, M}^{A, \eta_{1}}\left(A(x)+\rho_{1} f(x)-\rho_{1} F(z, y)\right), \\
y=(1-\lambda) y+\lambda R_{\rho_{2}, N}^{B, \eta_{2}}\left(B(y)+\rho_{2} g(y)-\rho_{2} G(w, x)\right),
\end{array}\right.
$$

where $\rho_{i}>0$ is a constant $(i=1,2)$;

Proof This directly follows from the definition of $R_{\rho_{1}, M}^{A, \eta_{1}}, R_{\rho_{2}, N}^{B, \eta_{2}}$, and the problem (3) for $i=1,2$.

Theorem 3.2 Let $X$ be a q-uniformly smooth Banach space. Let $f, g: X \rightarrow X$ be two singlevalued $\kappa_{1}$ or $\kappa_{2}$-Lipschitz continuous mappings, respectively, $\eta_{i}: X \times X \rightarrow X$ be a singlevalued $\tau_{i}$-Lipschitz continuous mapping $(i=1,2), F, G: X \times X \rightarrow X$ be two single-valued $\left(\mu_{1}, v_{1}\right)$ or $\left(\mu_{2}, v_{2}\right)$-Lipschitz continuous mappings, respectively. Let $A: X \rightarrow X$ be singlevalued $r_{1}$-strongly $\eta_{1}$-accretive, $\omega_{1}$-Lipschitz continuous, $\left(\sigma_{1}, \varphi_{1}\right)$-relaxed cocoercive with respect to $f$, and $B: X \rightarrow X$ be single-valued $r_{2}$-strongly $\eta_{2}$-accretive, $\omega_{2}$-Lipschitz continuous, $\left(\sigma_{2}, \varphi_{2}\right)$-relaxed cocoercive with respect to $g$. Let $S, T: X \rightarrow X$ be two set-valued $\gamma_{1}$ or $\gamma_{2}$-Lipschitz continuous mappings, respectively. If $M: X \rightarrow 2^{X}$ is a set-valued $\left(A, \eta_{1}\right)$ accretive mapping and $N: X \rightarrow 2^{X}$ is a set-valued $\left(B, \eta_{2}\right)$-accretive mapping, and the following condition holds:

$$
\left\{\begin{array}{lc}
\tau^{q}\left(\rho_{1} \mu_{1} \gamma_{1}+l_{1}\right)<\tau\left(r_{1}-m_{1} \rho_{1}\right), & \tau^{q}\left(\rho_{2} \mu_{2} \gamma_{2}+l_{2}\right)<\tau\left(r_{2}-m_{2} \rho_{2}\right), \\
l_{1}=\sqrt[q]{\omega_{1}^{q}+c_{q} \rho_{1}^{q} \kappa_{1}^{q}+q \sigma_{1} \omega_{1}^{q}-q \varphi_{1},} & l_{2}=\sqrt[q]{\omega_{2}^{q}+c_{q} \rho_{2}^{q} \kappa_{2}^{q}+q \sigma_{2} \omega_{2}^{q}-q \varphi_{2}},
\end{array}\right.
$$

where $c_{q}>0$ is the same as in Lemma 2.7 and $\rho_{i} \in\left(0, \frac{r_{i}}{m_{i}}\right)(i=1,2)$, then the problem (3) has a solution $x^{\prime \prime}, y^{\prime \prime} \in X, z^{\prime \prime} \in S\left(x^{\prime \prime}\right), w^{\prime \prime} \in T\left(y^{\prime \prime}\right)$.

Proof Define two mappings $Q_{1}, Q_{2}: X \rightarrow X$ as follows:

$$
\left\{\begin{array}{c}
Q_{1}(x)=(1-\lambda) x+\lambda R_{\rho_{1}, M}^{A, \eta_{1}}\left(A(x)+\rho_{1} f(x)-\rho_{1} F(z, y)\right), \\
Q_{2}(y)=(1-\lambda) y+\lambda R_{\rho_{2}, N}^{B, \eta_{2}}\left(B(y)+\rho_{2} g(y)-\rho_{2} G(w, x)\right) \\
\quad(\forall x, y \in X, z \in S(x), w \in T(y)) .
\end{array}\right.
$$

For elements $x_{1}, x_{2}, y_{1}, y_{2} \in X$, if letting

$$
\Omega_{i}=A\left(x_{i}\right)-\rho_{1} f\left(x_{i}\right)-\rho_{1} F\left(z_{i}, y_{i}\right) \quad(i=1,2),
$$


then by (8), Lemma 2.5 and Lemma 2.7, we have

$$
\begin{aligned}
\left\|Q_{1}\left(x_{1}\right)-Q_{1}\left(x_{2}\right)\right\|= & \left\|(1-\lambda) x_{1}+\lambda R_{\rho_{1}, M}^{A, \eta_{1}}\left(\Omega_{1}\right)-(1-\lambda) x_{2}-\lambda R_{\rho_{1}, M}^{A, \eta_{1}}\left(\Omega_{2}\right)\right\| \\
\leq & (1-\lambda)\left\|x_{1}-x_{2}\right\|+\lambda\left\|R_{\rho_{1}, M}^{A, \eta_{1}}\left(\Omega_{1}\right)-R_{\rho_{1}, M}^{A, \eta_{1}}\left(\Omega_{2}\right)\right\| \\
\leq & (1-\lambda)\left\|x_{1}-x_{2}\right\|+\lambda \frac{\tau^{q-1}}{r_{1}-m_{1} \rho_{1}}\left[\rho_{1}\left(\left\|F\left(z_{2}, y_{2}\right)-F\left(z_{1}, y_{1}\right)\right\|\right)\right. \\
& \left.+\left\|A\left(x_{1}\right)-A\left(x_{2}\right)-\rho_{1}\left(f\left(x_{1}\right)-f\left(x_{2}\right)\right)\right\|\right],
\end{aligned}
$$

and by $\left(\mu_{1}, v_{1}\right)$-Lipschitz continuity of $F(\cdot, \cdot)$ and $\gamma_{1}$-Lipschitz continuity of $S$, we obtain

$$
\begin{aligned}
\left\|F\left(z_{2}, y_{2}\right)-F\left(z_{1}, y_{1}\right)\right\| & \leq \mu_{1}\left\|z_{2}-z_{1}\right\|+v_{1}\left\|y_{2}-y_{1}\right\| \\
& \leq \mu_{1} \gamma_{1}\left\|x_{2}-x_{1}\right\|+v_{1}\left\|y_{2}-y_{1}\right\| .
\end{aligned}
$$

Since $A$ is $\omega_{1}$-Lipschitz continuous and $\left(\sigma_{1}, \varphi_{1}\right)$-relaxed cocoercive with respect to $f$, and $f$ is $\kappa_{1}$-Lipschitz continuous so that for $z_{1} \in S\left(x_{1}\right), z_{2} \in S\left(x_{2}\right)$, we have

$$
\begin{aligned}
& \left\|A\left(x_{1}\right)-A\left(x_{2}\right)-\rho_{1}\left(f\left(x_{1}\right)-f\left(x_{2}\right)\right)\right\|^{q} \\
& \leq\left\|A\left(x_{1}\right)-A\left(x_{2}\right)\right\|^{q}+c_{q} \rho_{1}^{q}\left\|f\left(x_{1}\right)-f\left(x_{2}\right)\right\|^{q} \\
& \quad-q\left\langle A\left(x_{1}\right)-A\left(x_{2}\right), J_{q}\left(f\left(x_{1}\right)-f\left(x_{2}\right)\right)\right\rangle \\
& \leq\left(\omega_{1}^{q}+c_{q} \rho_{1}^{q} \kappa_{1}^{q}+q \sigma_{1} \omega_{1}^{q}-q \varphi_{1}\right)\left\|x_{2}-x_{1}\right\|^{q} .
\end{aligned}
$$

Combining (9), (10) and (11), we can get

$$
\begin{aligned}
& \left\|Q_{1}\left(x_{1}\right)-Q_{1}\left(x_{2}\right)\right\| \\
& \leq(1-\lambda)\left\|x_{1}-x_{2}\right\|+\lambda \frac{\tau^{q-1}}{r_{1}-m_{1} \rho_{1}}\left[\rho_{1}\left(\mu_{1} \gamma_{1}\left\|x_{2}-x_{1}\right\|+v_{1}\left\|y_{2}-y_{1}\right\|\right)\right. \\
& \left.\quad+\sqrt[q]{\omega_{1}^{q}+c_{q} \rho_{1}^{q} \kappa_{1}^{q}+q \sigma_{1} \omega_{1}^{q}-q \varphi_{1}}\left(\omega_{1}^{q}+c_{q} \rho_{1}^{q} \kappa_{1}^{q}+q \sigma_{1} \omega_{1}^{q}-q \varphi_{1}\right)\left\|x_{2}-x_{1}\right\|\right] \\
& \leq\left((1-\lambda)+\lambda \theta_{1}\right)\left\|x_{1}-x_{2}\right\|+\lambda \frac{\tau^{q-1}}{r_{1}-m_{1} \rho_{1}} v_{1}\left\|y_{2}-y_{1}\right\|,
\end{aligned}
$$

where

$$
\theta_{1}=\frac{\tau^{q-1}}{r_{1}-m_{1} \rho_{1}}\left(\rho_{1} \mu_{1} \gamma_{1}+\sqrt[q]{\omega_{1}^{q}+c_{q} \rho_{1}^{q} \kappa_{1}^{q}+q \sigma_{1} \omega_{1}^{q}-q \varphi_{1}}\right) .
$$

For elements $x_{1}, x_{2}, y_{1}, y_{2} \in X, z_{i} \in S\left(x_{i}\right), y_{i} \in T\left(y_{i}\right)(i=1,2)$, if letting

$$
\Theta_{i}=B\left(y_{i}\right)+\rho_{2} g\left(y_{i}\right)-\rho_{2} G\left(w_{i}, x_{i}\right) \quad(i=1,2),
$$

then by using the same method as the one used above,

$$
\begin{aligned}
\left\|Q_{2}\left(y_{1}\right)-Q_{2}\left(y_{2}\right)\right\| & =\left\|(1-\lambda) y_{1}+\lambda R_{\rho_{2}, N}^{B, \eta_{2}}\left(\Theta_{1}\right)-(1-\lambda) y_{2}-\lambda R_{\rho_{2}, N}^{B, \eta_{2}}\left(\Theta_{2}\right)\right\| \\
& \leq(1-\lambda)\left\|y_{1}-y_{2}\right\|+\lambda\left\|R_{\rho_{2}, N}^{B, \eta_{2}}\left(\Theta_{1}\right)-R_{\rho_{2}, N}^{B, \eta_{2}}\left(\Theta_{2}\right)\right\|
\end{aligned}
$$




$$
\begin{aligned}
\leq & (1-\lambda)\left\|y_{1}-y_{2}\right\|+\lambda \frac{\tau^{q-1}}{r_{2}-m_{2} \rho_{2}}\left(\rho_{2}\left\|G\left(w_{2}, x_{2}\right)-G\left(w_{1}, x_{1}\right)\right\|\right. \\
& \left.+\left\|B\left(y_{1}\right)-B\left(y_{2}\right)-\rho_{2}\left(g\left(y_{1}\right)-g\left(y_{2}\right)\right)\right\|\right) \\
\leq & \lambda \frac{\tau^{q-1}}{r_{2}-m_{2} \rho_{2}} \rho_{2} v_{2}\left\|x_{2}-x_{1}\right\|+\left[(1-\lambda)+\lambda \theta_{2}\right]\left\|y_{2}-y_{1}\right\|
\end{aligned}
$$

hold, where

$$
\theta_{2}=\frac{\tau^{q-1}}{r_{2}-m_{2} \rho_{2}}\left(\rho_{2} \mu_{2} \gamma_{2}+\sqrt[q]{\omega_{2}^{q}+c_{q} \rho_{2}^{q} \kappa_{2}^{q}+q \sigma_{2} \omega_{2}^{q}-q \varphi_{2}}\right) .
$$

If setting

$$
\begin{aligned}
& \Gamma_{11}=\theta_{1}, \quad \Gamma_{12}=\frac{\tau^{q-1}}{r_{1}-m_{1} \rho_{1}} \rho_{1} v_{1}, \\
& \Gamma_{21}=\frac{\tau^{q-1}}{r_{2}-m_{2} \rho_{2}} \rho_{2} v_{2}, \quad \Gamma_{22}=\theta_{2},
\end{aligned}
$$

$\vec{a}=\left(\left\|Q_{1}\left(x_{1}\right)-Q_{1}\left(x_{2}\right)\right\|,\left\|Q_{2}\left(y_{1}\right)-Q_{2}\left(y_{2}\right)\right\|\right)^{T}$ and $\vec{b}=\left(\left\|x_{1}-x_{2}\right\|,\left\|y_{1}-y_{2}\right\|\right)^{T}$, then from (12), (13) and (14), we have $\vec{a} \leq(1-\lambda) \mathbf{E}+\lambda \boldsymbol{\Psi} \vec{b}$, where

$$
\mathbf{E}=\left(\begin{array}{ll}
1 & 0 \\
0 & 1
\end{array}\right), \quad \boldsymbol{\Psi}=\left(\begin{array}{ll}
\Gamma_{11} & \Gamma_{12} \\
\Gamma_{21} & \Gamma_{22}
\end{array}\right), \quad 0<\lambda<1,
$$

where $\boldsymbol{\Psi}$ is called the matrix for nonlinear set-valued inclusions system. By using [16], we have

$$
\|\vec{a}\| \leq(1-\lambda)+\lambda\|\Psi\|\|\vec{b}\|
$$

Letting

$$
\|\Psi\|=\max \left\{\Gamma_{11}, \Gamma_{12}, \Gamma_{21}, \Gamma_{22}\right\}
$$

It follows from (16), the assumption of the condition (7) and $S(x), T(y) \in C B(X)$ that $0<\|\Psi\|<1,(1-\lambda)+\lambda\|\Psi\|<1$, and there exist $x^{*}, y^{*} \in X$ and $z^{*} \in S\left(x^{*}\right), w^{*} \in T\left(y^{*}\right)$ such that

$$
\left\{\begin{array}{l}
Q_{1}\left(x^{*}\right)=x^{*} \\
Q_{2}\left(y^{*}\right)=y^{*}
\end{array}\right.
$$

Therefore, the following relations hold for Theorem 3.1(ii)-(iii):

$$
\left\{\begin{array}{l}
x^{*}=R_{\rho_{1}, M}^{A, \eta_{1}}\left(A\left(x^{*}\right)+\rho_{1} f\left(x^{*}\right)-\rho_{1} F\left(z^{*}, y^{*}\right)\right), \\
y^{*}=R_{\rho_{2}, N}^{B, \eta_{2}}\left(B\left(y^{*}\right)+\rho_{2} g\left(y^{*}\right)-\rho_{2} G\left(w^{*}, x^{*}\right)\right),
\end{array}\right.
$$

where $\rho_{i}>0$ is a constant $(i=1,2)$. Thus, by Theorem 3.1 , we know that $\left(x^{*}, y^{*}, z^{*}, w^{*}\right)$ is a solution of the problem (3). This completes the proof. 


\section{Ishikawa-hybrid proximal algorithm}

In 2008, Verma developed a hybrid version of the Eckstein-Bertsekas [11] proximal point algorithm, introduced the algorithm based on the $(A, \eta)$-maximal monotonicity framework [7] and studied convergence of the algorithm, and so did Li, Xu and Jin in [12]. Based on Theorem 3.1, we develop an Ishikawa-hybrid proximal point algorithm for finding an iterative sequence solving the problem (3) as follows.

Algorithm 4.1 Let $X$ be a $q$-uniformly smooth Banach space. Let $f, g: X \rightarrow X$ be two single-valued $\kappa_{1}$ or $\kappa_{2}$-Lipschitz continuous mappings, respectively, $\eta_{i}: X \times X \rightarrow X$ be a single-valued $\tau_{i}$-Lipschitz continuous mapping $(i=1,2), F, G: X \times X \rightarrow X$ be two singlevalued $\left(\mu_{1}, v_{1}\right)$ or $\left(\mu_{2}, v_{2}\right)$-Lipschitz continuous mappings, respectively. Let $A: X \rightarrow X$ be single-valued $r_{1}$-strongly $\eta_{1}$-accretive, $\omega_{1}$-Lipschitz continuous, $\left(\sigma_{1}, \varphi_{1}\right)$-relaxed cocoercive with respect to $f$, and $B: X \rightarrow X$ be single-valued $r_{2}$-strongly $\eta_{2}$-accretive, $\omega_{2}$ Lipschitz continuous, $\left(\sigma_{2}, \varphi_{2}\right)$-relaxed cocoercive with respect to $g$. Let $S, T: X \rightarrow X$ be two set-valued $\gamma_{1}$ or $\gamma_{2}$-Lipschitz continuous mappings, respectively, $M: X \rightarrow 2^{X}$ be a setvalued $\left(A, \eta_{1}\right)$-accretive mapping and $N: X \rightarrow 2^{X}$ be a set-valued $\left(B, \eta_{2}\right)$-accretive mapping. Suppose that $\left\{\alpha_{i}^{n}\right\}_{n=0}^{\infty},\left\{\beta_{i}^{n}\right\}_{n=0}^{\infty},\left\{\xi_{i}^{n}\right\}_{n=0}^{\infty},\left\{\zeta_{i}^{n}\right\}_{n=0}^{\infty}$ and $\left\{\rho_{i}^{n}\right\}_{n=0}^{\infty}(i=1,2)$ are ten nonnegative sequences such that

$$
\begin{aligned}
& \lim _{n \rightarrow \infty} \xi_{i}^{n}=0, \quad \lim _{n \rightarrow \infty} \zeta_{i}^{n}=0, \quad \alpha=\limsup _{n \rightarrow \infty} \alpha_{i}^{n}<1, \\
& \beta=\limsup _{n \rightarrow \infty} \beta_{i}^{n}<1, \quad \rho_{i}^{n} \uparrow \rho_{i}<\frac{r_{i}}{m_{i}} \quad(i=1,2),
\end{aligned}
$$

then we can get $x^{1}, y^{1} \in X$ and $z^{1} \in S\left(x^{1}\right), w^{1} \in T\left(y^{1}\right)$ as follows.

Step 1: For arbitrarily chosen initial points $x^{0} \in X, y^{0} \in X$, we choose suitable $z^{0} \in S\left(x^{0}\right)$, $w^{0} \in T\left(y^{0}\right)$, setting

$$
\left\{\begin{array}{l}
u^{0}=\left(1-\alpha_{1}^{0}\right) x^{0}+\alpha_{1}^{0} e_{1}^{0}, \\
x^{1}=\left(1-\beta_{1}^{0}\right) x^{0}+\beta_{1}^{0} d_{1}^{0},
\end{array}\right.
$$

where $e_{1}^{0}, d_{1}^{0}$ satisfy

$$
\left\{\begin{array}{l}
\left\|e_{1}^{0}-R_{\rho_{1}^{0}, M}^{A, \eta_{1}}\left(A\left(x^{0}\right)+\rho_{1}^{0} f\left(x^{0}\right)+\rho_{1}^{0} F\left(z^{0}, y^{0}\right)\right)\right\| \\
\quad \leq \xi_{1}^{0}\left\|e_{1}^{0}-x^{0}\right\| \quad\left(z^{0} \in S\left(x^{0}\right)\right) \\
\left\|d_{1}^{0}-R_{\rho_{1}^{0}, M}^{A, \eta_{1}}\left(A\left(u^{0}\right)+\rho_{1}^{0} f\left(u^{0}\right)+\rho_{1}^{0} F\left(z_{1}^{0}, y^{0}\right)\right)\right\| \\
\leq \zeta_{1}^{0}\left\|d_{1}^{0}-u^{0}\right\| \quad\left(z_{1}^{0} \in S\left(u^{0}\right)\right)
\end{array}\right.
$$

and

$$
\left\{\begin{array}{l}
v^{0}=\left(1-\alpha_{2}^{0}\right) y^{0}+\alpha_{2}^{0} e_{2}^{0}, \\
y^{1}=\left(1-\beta_{2}^{0}\right) y^{0}+\beta_{2}^{0} d_{2}^{0},
\end{array}\right.
$$


where $e_{2}^{0}, d_{2}^{0}$ satisfy

$$
\left\{\begin{array}{l}
\left\|e_{2}^{0}-R_{\rho_{2}^{0}, N}^{B, \eta_{2}}\left(B\left(y^{0}\right)+\rho_{2}^{0} g\left(y^{0}\right)-\rho_{2}^{0} G\left(w^{0}, x^{0}\right)\right)\right\| \\
\quad \leq \xi_{2}^{0}\left\|e_{2}^{0}-y^{0}\right\| \quad\left(w^{0} \in T\left(y^{0}\right),\right. \\
\left\|d_{2}^{0}-R_{\rho_{2}^{0}, N}^{B, \eta_{2}}\left(B\left(v^{0}\right)+\rho_{2}^{0} g\left(v^{0}\right)-\rho_{2}^{0} G\left(w_{2}^{0}, x^{0}\right)\right)\right\| \\
\leq \zeta_{2}^{0}\left\|d_{2}^{0}-v^{0}\right\| \quad\left(w_{2}^{0} \in T\left(v^{0}\right)\right) .
\end{array}\right.
$$

By using Nadler [15], we can choose suitable $z^{1} \in S\left(x^{1}\right), w^{1} \in T\left(y^{1}\right)$ such that

$$
\left\{\begin{array}{l}
\left\|z^{0}-z^{1}\right\| \leq\left(1+\frac{1}{1}\right) D\left(S\left(x^{0}\right), S\left(x^{1}\right)\right), \\
\left\|w^{0}-w^{1}\right\| \leq\left(1+\frac{1}{1}\right) D\left(T\left(y^{0}\right), T\left(y^{1}\right)\right), \\
\left\|z_{1}^{0}-z_{1}^{1}\right\| \leq\left(1+\frac{1}{1}\right) D\left(S\left(U^{0}\right), S\left(U^{1}\right)\right), \\
\left\|w_{2}^{0}-w_{2}^{1}\right\| \leq\left(1+\frac{1}{1}\right) D\left(T\left(V^{0}\right), T\left(V^{1}\right)\right) .
\end{array}\right.
$$

Therefore, we obtain $x^{1}, y^{1} \in X$ and $z^{1} \in S\left(x^{1}\right), w^{1} \in T\left(y^{1}\right)$ and give the next step for generating sequences $\left\{x^{n}\right\}_{n=2}^{\infty},\left\{y^{n}\right\}_{n=2}^{\infty},\left\{z^{n}\right\}_{n=2}^{\infty}$ and $\left\{w^{n}\right\}_{n=2}^{\infty}$.

Step 2: From $x^{1}, y^{1} \in X$ and $z^{1} \in S\left(x^{1}\right), w^{1} \in T\left(y^{1}\right)$, the sequences $\left\{x^{n}\right\}_{n=2}^{\infty},\left\{y^{n}\right\}_{n=2}^{\infty},\left\{z^{n}\right\}_{n=2}^{\infty}$ and $\left\{w^{n}\right\}_{n=2}^{\infty}$ are generated by the iterative procedure

$$
\left\{\begin{array}{l}
u^{n}=\left(1-\alpha_{1}^{n}\right) x^{n}+\alpha_{1}^{n} e_{1}^{n}, \\
x^{n+1}=\left(1-\beta_{1}^{n}\right) x^{n}+\beta_{1}^{n} d_{1}^{n}, \\
\left\|e_{1}^{n}-R_{\rho_{1}^{n}, M}^{A, \eta_{1}}\left(A\left(x^{n}\right)+\rho_{1}^{n} f\left(x^{n}\right)+\rho_{1}^{n} F\left(z^{n}, y^{n}\right)\right)\right\| \\
\quad \leq \xi_{1}^{n}\left\|e_{1}^{n}-x^{n}\right\| \quad\left(z^{n} \in S\left(x^{n}\right)\right), \\
\left\|d_{1}^{n}-R_{\rho_{1}^{n}, M}^{A, \eta_{1}}\left(A\left(u^{n}\right)+\rho_{1}^{n} f\left(u^{n}\right)-\rho_{1}^{n} F\left(z_{1}^{n}, y^{n}\right)\right)\right\| \\
\quad \leq \zeta_{1}^{n}\left\|d_{1}^{n}-u^{n}\right\| \quad\left(z_{1}^{n} \in S\left(u^{n}\right)\right),
\end{array}\right.
$$

and

$$
\left\{\begin{array}{l}
v^{n}=\left(1-\alpha_{2}^{n}\right) y^{n}+\alpha_{2}^{n} e_{2}^{n}, \\
y^{n+1}=\left(1-\beta_{2}^{n}\right) y^{n}+\beta_{2}^{n} d_{2}^{n}, \\
\left\|e_{2}^{n}-R_{\rho_{2}^{n}, N}^{B, \eta_{2}}\left(B\left(y^{n}\right)+\rho_{2}^{n} g\left(y^{n}\right)-\rho_{2}^{n} G\left(w^{n}, x^{n}\right)\right)\right\| \\
\quad \leq \xi_{2}^{n}\left\|e_{2}^{n}-y^{n}\right\| \quad\left(w^{n} \in T\left(y^{n}\right)\right), \\
\left\|d_{2}^{n}-R_{\rho_{2}^{n}, N}^{B, \eta_{2}}\left(B\left(v^{n}\right)+\rho_{2}^{n} g\left(v^{n}\right)-\rho_{2}^{n} G\left(w_{2}^{n}, x^{n}\right)\right)\right\| \\
\quad \leq \zeta_{2}^{n}\left\|d_{2}^{n}-v^{n}\right\| \quad\left(w_{2}^{n} \in T\left(v^{n}\right)\right) .
\end{array}\right.
$$

By using Nadler [15], we can choose suitable $z^{n+1} \in S\left(x^{n+1}\right), w^{n+1} \in T\left(y^{n+1}\right)$ such that

$$
\left\{\begin{array}{l}
\left\|z^{n}-z^{n+1}\right\| \leq\left(1+\frac{1}{1+n}\right) D\left(S\left(x^{n}\right), S\left(x^{n+1}\right)\right), \\
\left\|w^{n}-w^{n+1}\right\| \leq\left(1+\frac{1}{1+n}\right) D\left(T\left(y^{n}\right), T\left(y^{n+1}\right)\right),
\end{array}\right.
$$

for $n=0,1,2, \ldots$ 
Remark 4.2 If we choose some suitable operators $A, B, \eta_{1}, \eta_{1}, F, G, S, T, M, N, f, g$ and a space $X$, then Algorithm 4.1 can degenerate to a number of known algorithms for solving the system of variational inequalities and variational inclusions (see $[2-6,8-10,25]$ ).

\section{Convergence of Ishikawa-hybrid proximal Algorithm 4.1}

In this section, we prove that $\left\{\left(x^{n}, y^{n}, z^{n}, w^{n}\right)\right\}_{n=0}^{\infty}$ generated by Ishikawa-hybrid proximal Algorithm 4.1 converges linearly to a solution $\left(x^{*}, y^{\prime \prime}, z^{*}, w^{*}\right)$ of the problem (3) as the convergence rate $\|\boldsymbol{\Psi}\|$.

Theorem 5.1 Let $X$ be a q-uniformly smooth Banach space. Let $f, g: X \rightarrow X$ be two single-valued $\kappa_{1}$ or $\kappa_{2}$-Lipschitz continuous mappings, respectively, $\eta_{i}: X \times X \rightarrow X$ be a single-valued $\tau_{i}$-Lipschitz continuous mapping $(i=1,2), F, G: X \times X \rightarrow X$ be two singletwo valued $\left(\mu_{1}, v_{1}\right)$ or $\left(\mu_{2}, v_{2}\right)$-Lipschitz continuous mappings, respectively. Let $A: X \rightarrow X$ be a single-valued $r_{1}$-strongly $\eta_{1}$-accretive and $\omega_{1}$-Lipschitz continuous mapping, and let $B: X \rightarrow X$ be a single-valued $r_{2}$-strongly $\eta_{2}$-accretive and $\omega_{2}$-Lipschitz continuous mapping. Let $S, T: X \rightarrow X$ be two set-valued $\gamma_{1}$ or $\gamma_{2}$-Lipschitz continuous mappings, respectively, $A$ be $\left(\sigma_{1}, \varphi_{1}\right)$-relaxed cocoercive with respect to $f$ and $B$ be $\left(\sigma_{2}, \varphi_{2}\right)$-relaxed cocoercive with respect to $g$. Suppose that $M: X \rightarrow 2^{X}$ is a set-valued $\left(A, \eta_{1}\right)$-accretive mapping and $N: X \rightarrow 2^{X}$ is a set-valued $\left(B, \eta_{2}\right)$-accretive mapping, and the following conditions hold:

$$
\left\{\begin{array}{l}
\max \left\{\rho_{1} \mu_{1} \gamma_{1}+l_{1}, \alpha_{1} \beta_{1} v_{1} \theta_{1}\left(1+\rho_{1}-\beta_{1} \rho_{1}\right),\right. \\
\left.\quad \beta_{2} \rho_{2} v_{2}\left(1+2 \alpha_{2} \theta_{2}\right),\left(\rho_{2} \mu_{2} \gamma_{2}+l_{2}\right)\right\}<\tau^{1-q}\left(r_{1}-m_{1} \rho_{1}\right), \\
2 \frac{\beta_{1}\left(1-\alpha_{1}\right)}{\alpha_{1}+\beta_{1}} \theta_{1}+\frac{\alpha_{1} \beta_{1}}{\alpha_{1}+\beta_{1}} \theta_{1}^{2}<1-\theta_{1}, \\
\left(1-\beta_{2}+\beta_{2} \theta_{2}\right)+3\left(1-\alpha_{2}+\alpha_{2} \theta_{2}\right) \beta_{2} \theta_{2}+\beta_{2} \theta_{2}<1 \\
l_{1}=\sqrt[q]{\omega_{1}^{q}+c_{q} \rho_{1}^{q} \kappa_{1}^{q}+q \sigma_{1} \omega_{1}^{q}-q \varphi_{1}}, \quad l_{2}=\sqrt[q]{\omega_{2}^{q}+c_{q} \rho_{2}^{q} \kappa_{2}^{q}+q \sigma_{2} \omega_{2}^{q}-q \varphi_{2}} \\
\theta_{1}=\frac{\tau^{q-1}}{r_{1}-m_{1} \rho_{1}}\left(\rho_{1} \mu_{1} \gamma_{1}+l_{1}\right), \quad \theta_{2}=\frac{{ }_{\tau^{q-1}}-m_{2} \rho_{2}}{r_{2}}\left(\rho_{2} \mu_{2} \gamma_{2}+l_{2}\right),
\end{array}\right.
$$

and eight nonnegative sequences $\left\{\alpha_{i}^{n}\right\}_{n=0}^{\infty},\left\{\beta_{i}^{n}\right\}_{n=0}^{\infty},\left\{\xi_{i}^{n}\right\}_{n=0}^{\infty},\left\{\zeta_{i}^{n}\right\}_{n=0}^{\infty}$ and $\left\{\rho_{i}^{n}\right\}_{n=0}^{\infty}(i=1,2)$ satisfy the following conditions:

$$
\begin{aligned}
& \lim _{n \rightarrow \infty} \xi_{i}^{n}=\lim _{n \rightarrow \infty} \zeta_{i}^{n}=0, \quad \alpha_{i}=\limsup _{n \rightarrow \infty} \alpha_{i}^{n}<1, \\
& \beta_{i}=\limsup _{n \rightarrow \infty} \beta_{i}^{n}<1, \quad \rho_{i}^{n} \uparrow \rho_{i}<\frac{r_{i}}{m_{i}} .
\end{aligned}
$$

Then the problem (3) has a solution $\left(x^{\prime \prime}, y^{\prime \prime}, z^{\prime \prime}, w^{\prime \prime}\right) z^{\prime \prime} \in S\left(x^{\prime \prime}\right), w^{\prime \prime} \in T\left(y^{\prime \prime}\right)$, and the sequence $\left\{x^{n}, y^{n}\right\}_{n=0}^{\infty}$ generated by Ishikawa-hybrid proximal Algorithm 4.1 converges linearly to a solution $\left(x^{*}, y^{*}\right)$ of the problem (3) as the convergence rate

$$
\begin{aligned}
\|\boldsymbol{\Psi}\|= & \max \left\{1-\left(\alpha_{1}+\beta_{1}\right)+\left(\alpha_{1}+\beta_{1}\right) \theta_{1}+\left(2-2 \alpha_{1}+\alpha_{1} \theta_{1}\right) \beta_{1} \theta_{1},\right. \\
& \alpha_{1} \beta_{1} v_{1} \theta_{1} \frac{\tau^{q-1}}{r_{1}-m_{1} \rho_{1}}\left(1+\rho_{1}-\beta_{1} \rho_{1}\right), \\
& 1-\beta_{2}+2 \beta_{2} \theta_{2}\left(2-\alpha_{2}+\alpha_{2} \theta_{2}\right)+\beta_{2} \theta_{2}\left(1-\alpha_{2}+\alpha_{2} \theta_{2}\right), \\
& \left.\left(1-\beta_{2}+\beta_{2} \theta_{2}\right)+3\left(1-\alpha_{2}+\alpha_{2} \theta_{2}\right) \beta_{2} \theta_{2}+\beta_{2} \theta_{2}\right\}
\end{aligned}
$$

where $c_{q}>0$ is the same as in Lemma 2.5, $\rho_{i} \in\left(0, \frac{r_{i}}{m_{i}}\right)(i=1,2)$. 
Proof Let $\left(x^{*}, y^{*}, z^{*}, w^{*}\right)\left(z^{*} \in S\left(x^{*}\right), w^{\prime \prime} \in T\left(y^{*}\right)\right)$ be the solution of the problem (3), then for any $\lambda>0$,

$$
\left\{\begin{array}{l}
x^{*}=(1-\lambda) x^{*}+\lambda R_{\rho_{1}, M}^{A, \eta_{1}}\left(A\left(x^{*}\right)+\rho_{1} f\left(x^{*}\right)-\rho_{1} F\left(z^{*}, y^{*}\right)\right), \\
y^{*}=(1-\lambda) y^{*}+\lambda R_{\rho_{2}, N}^{B, \eta_{2}}\left(B\left(y^{*}\right)+\rho_{2} g\left(y^{*}\right)-\rho_{2} G\left(w^{*}, x^{*}\right)\right) .
\end{array}\right.
$$

For $n \geq 0$, we write

$$
\left\{\begin{array}{l}
s_{1}^{n}=\left(1-\alpha_{1}^{n}\right) x^{n}+\alpha_{1}^{n} R_{\rho_{1}^{n}, M}^{A, \eta_{1}}\left(A\left(x^{n}\right)+\rho_{1}^{n} f\left(x^{n}\right)+\rho_{1}^{n} F\left(z^{n}, y^{n}\right)\right), \\
t_{1}^{n+1}=\left(1-\beta_{1}^{n}\right) x^{n}+\beta_{1}^{n} R_{\rho_{1}^{n}, M}^{A, \eta_{1}}\left(A\left(s_{1}^{n}\right)+\rho_{1}^{n} f\left(s_{1}^{n}\right)+\rho_{1}^{n} F\left(z_{2}^{n}, y^{n}\right)\right) \\
\quad\left(z_{2}^{n} \in S\left(s_{1}^{n}\right)\right) .
\end{array}\right.
$$

It follows from the hypotheses of the mappings $A, f, F, S, M, \eta_{1}$ and $R_{\rho_{1}^{n}, M}^{A, \eta_{1}}$ in Algorithm 4.1 that

$$
\begin{aligned}
& \left\|s_{1}^{n}-x^{*}\right\| \leq \|\left(1-\alpha_{1}^{n}\right) x^{n}+\alpha_{1}^{n} R_{\rho_{1}^{n}, M}^{A, \eta_{1}}\left(A\left(x^{n}\right)+\rho_{1}^{n} f\left(x^{n}\right)+\rho_{1}^{n} F\left(z^{n}, y^{n}\right)\right) \\
& -\left(1-\alpha_{1}^{n}\right) x^{*}-\alpha_{1}^{n} R_{\rho_{1}^{n}, M}^{A, \eta_{1}}\left(A\left(x^{*}\right)+\rho_{1}^{n} f\left(x^{*}\right)-\rho_{1}^{n} F\left(z^{*}, y^{*}\right)\right) \| \\
& \leq\left[\left(1-\alpha_{1}^{n}\right)+\alpha_{1}^{n} \theta_{1}\right]\left\|x^{n}-x^{*}\right\|+\alpha_{1}^{n} \frac{\tau^{q-1}}{r_{1}-m_{1} \rho_{1}^{n}} \rho_{1}^{n} \nu_{1}\left\|y^{n}-y^{*}\right\|, \\
& \left\|u^{n}-x^{*}\right\| \leq \|\left(1-\alpha_{1}^{n}\right) x^{n}+\alpha_{1}^{n} e_{1}^{n} \\
& \text { - }\left(1-\alpha_{1}^{n}\right) x^{*}-\alpha_{1}^{n} R_{\rho_{1}^{n}, M}^{A, \eta_{1}}\left(A\left(x^{*}\right)+\rho_{1}^{n} f\left(x^{*}\right)-\rho_{1}^{n} F\left(z^{*}, y^{*}\right)\right) \| \\
& \leq\left(1-\alpha_{1}^{n}\right)\left\|x^{n}-x^{*}\right\|+\alpha_{1}^{n}\left\|e_{1}^{n}-R_{\rho_{1}^{n}, M}^{A, \eta_{1}}\left(A\left(x^{*}\right)+\rho_{1}^{n} f\left(x^{*}\right)-\rho_{1}^{n} F\left(z^{*}, y^{*}\right)\right)\right\| \\
& \leq\left(1-\alpha_{1}^{n}\right)\left\|x^{n}-x^{*}\right\|+\alpha_{1}^{n}\left\|e_{1}^{n}-R_{\rho_{1}^{n}, M}^{A, \eta_{1}}\left(A\left(x^{n}\right)+\rho_{1}^{n} f\left(x^{n}\right)-\rho_{1}^{n} F\left(z^{n}, y^{n}\right)\right)\right\| \\
& +\alpha_{1}^{n} \| R_{\rho_{1}^{n}, M}^{A, \eta_{1}}\left(A\left(x^{n}\right)+\rho_{1}^{n} f\left(x^{n}\right)-\rho_{1}^{n} F\left(z^{n}, y^{n}\right)\right) \\
& -R_{\rho_{1}^{n}, M}^{A, \eta_{1}}\left(A\left(x^{*}\right)+\rho_{1}^{n} f\left(x^{*}\right)-\rho_{1}^{n} F\left(z^{*}, y^{*}\right)\right) \| \\
& \leq\left(1-\alpha_{1}^{n}\right)\left\|x^{n}-x^{*}\right\|+\alpha_{1}^{n} \xi_{1}^{n}\left\|e_{1}^{n}-x^{n}\right\| \\
& +\alpha_{1}^{n} \frac{\tau^{q-1}}{r_{1}-m_{1} \rho_{1}^{n}}\left[\rho_{1}^{n} \mu_{1} \gamma_{1}\left\|x^{n}-x^{*}\right\|+v_{1}\left\|y^{n}-y^{*}\right\|\right. \\
& \left.+\sqrt[q]{\omega_{1}^{q}+c_{q}\left(\rho_{1}^{n}\right)^{q} \kappa_{1}^{q}+q \sigma_{1} \omega_{1}^{q}-q \varphi_{1}}\left\|x^{n}-x^{*}\right\|\right] \\
& \leq\left(1-\alpha_{1}^{n}\right)\left\|x^{n}-x^{*}\right\|+\xi_{1}^{n}\left\|u^{n}-x^{*}\right\|+\xi_{1}^{n}\left\|x^{n}-x^{*}\right\| \\
& +\alpha_{1}^{n} \frac{\tau^{q-1}}{r_{1}-m_{1} \rho_{1}^{n}}\left[\rho_{1}^{n} \mu_{1} \gamma_{1}\left\|x^{n}-x^{*}\right\|+v_{1}\left\|y^{n}-y^{*}\right\|\right. \\
& \left.+\sqrt[q]{\omega_{1}^{q}+c_{q}\left(\rho_{1}^{n}\right)^{q} \kappa_{1}^{q}+q \sigma_{1} \omega_{1}^{q}-q \varphi_{1}}\left\|x^{n}-x^{*}\right\|\right] \\
& \leq\left(1-\alpha_{1}^{n}+\xi_{1}^{n}+\alpha_{1}^{n} \theta_{1}\right)\left\|x^{n}-x^{*}\right\|+\xi_{1}^{n}\left\|u^{n}-x^{*}\right\| \\
& +\alpha_{1}^{n} \frac{\tau^{q-1}}{r_{1}-m_{1} \rho_{1}^{n}} v_{1}\left\|y^{n}-y^{*}\right\| \text {; }
\end{aligned}
$$


that is,

$$
\begin{aligned}
\left\|s_{1}^{n}-x^{*}\right\| \leq & {\left[\left(1-\alpha_{1}^{n}\right)+\alpha_{1}^{n} \theta_{1}(n)\right]\left\|x^{n}-x^{*}\right\|+\alpha_{1}^{n} \frac{\tau^{q-1}}{r_{1}-m_{1} \rho_{1}^{n}} \rho_{1}^{n} \nu_{1}\left\|y^{n}-y^{*}\right\|, } \\
\left\|u^{n}-x^{*}\right\| \leq & \frac{1}{1-\xi_{1}^{n}}\left(1-\alpha_{1}^{n}+\xi_{1}^{n}+\alpha_{1}^{n} \theta_{1}(n)\right)\left\|x^{n}-x^{*}\right\| \\
& +\frac{1}{1-\xi_{1}^{n}} \alpha_{1}^{n} \frac{\tau^{q-1}}{r_{1}-m_{1} \rho_{1}^{n}} v_{1}\left\|y^{n}-y^{*}\right\|,
\end{aligned}
$$

where $\theta_{1}(n)=\frac{\tau^{q-1}}{r_{1}-m_{1} \rho_{1}^{n}}\left(\rho_{1}^{n} \mu_{1} \gamma_{1}+\sqrt[q]{\omega_{1}^{q}+c_{q}\left(\rho_{1}^{n}\right)^{q} \kappa_{1}^{q}+q \sigma_{1} \omega_{1}^{q}-q \varphi_{1}}\right), z^{n} \in S\left(x^{n}\right)$ and $x^{*} \in S\left(x^{*}\right)$.

From (24)-(27) and (13), we have

$$
\begin{aligned}
\left\|t_{1}^{n+1}-x^{*}\right\| \leq & \left(1-\beta_{1}^{n}\right)\left\|x^{n}-x^{*}\right\| \\
& +\beta_{1}^{n} \| R_{\rho_{1}^{n}, M}^{A, \eta_{1}}\left(A\left(x^{*}\right)+\rho_{1}^{n} f\left(x^{*}\right)-\rho_{1}^{n} F\left(z^{*}, y^{*}\right)\right) \\
& -R_{\rho_{1}^{n}, M}^{A, \eta_{1}}\left(A\left(s_{1}^{n}\right)+\rho_{1}^{n} f\left(s_{1}^{n}\right)+\rho_{1}^{n} F\left(z_{2}^{n}, y^{n}\right)\right) \| \\
\leq & \left(\left(1-\beta_{1}^{n}\right)+\beta_{1}^{n} \frac{\tau^{q-1}}{r_{1}-m_{1} \rho_{1}^{n}}\left(\rho_{1}^{n} \mu_{1} \gamma_{1}\right.\right. \\
& \left.+\sqrt[q]{\left.\omega_{1}^{q}+c_{q}\left(\rho_{1}^{n}\right)^{q} \kappa_{1}^{q}+q \sigma_{1} \omega_{1}^{q}-q \varphi_{1}\right)}\right)\left\|s_{1}^{n}-x^{*}\right\| \\
& +\beta_{1}^{n} \frac{\tau^{q-1}}{r_{1}-m_{1} \rho_{1}^{n}} v_{1}\left\|y^{n}-y^{*}\right\| \\
\leq & \left(\left(1-\beta_{1}^{n}\right)+\beta_{1}^{n} \theta_{1}(n)\right)\left\|s_{1}^{n}-x^{*}\right\|+\beta_{1}^{n} \frac{\tau^{q-1}}{r_{1}-m_{1} \rho_{1}^{n}} v_{1}\left\|y^{n}-y^{*}\right\| .
\end{aligned}
$$

By Algorithm 4.1, $x^{n+1}-x^{n}=\beta_{1}^{n}\left(d_{1}^{n}-x^{n}\right)$ and $u^{n}-x^{n}=\alpha_{1}^{n}\left(e_{1}^{n}-x^{n}\right)$, we have

$$
\begin{aligned}
\left\|x^{n+1}-t_{1}^{n+1}\right\| \leq & \|\left(1-\beta_{1}^{n}\right) x^{n}+\beta_{1}^{n} d_{1}^{n}-\left(1-\beta_{1}^{n}\right) x^{n} \\
& -\beta_{1}^{n} R_{\rho_{1}^{n}, M}^{A, \eta_{1}}\left(A\left(s_{1}^{n}\right)+\rho_{1}^{n} f\left(s_{1}^{n}\right)-\rho_{1}^{n} F\left(z_{2}^{n}, y^{n}\right)\right) \| \\
\leq & \beta_{1}^{n}\left\|d_{1}^{n}-R_{\rho_{1}^{n}, M}^{A, \eta_{1}}\left(A\left(s_{1}^{n}\right)+\rho_{1}^{n} f\left(s_{1}^{n}\right)-\rho_{1}^{n} F\left(z_{2}^{n}, y^{n}\right)\right)\right\| \\
\leq & \beta_{1}^{n}\left(\left\|d_{1}^{n}-R_{\rho_{1}^{n}, M}^{A, \eta_{1}}\left(A\left(u^{n}\right)+\rho_{1}^{n} f\left(u^{n}\right)-\rho_{1}^{n} F\left(z_{1}^{n}, y^{n}\right)\right)\right\|\right. \\
& +\| R_{\rho_{1}^{n}, M}^{A, \eta_{1}}\left(A\left(u^{n}\right)+\rho_{1}^{n} f\left(u^{n}\right)-\rho_{1}^{n} F\left(z_{1}^{n}, y^{n}\right)\right) \\
& \left.-R_{\rho_{1}^{n}, M}^{A, \eta_{1}}\left(A\left(s_{1}^{n}\right)+\rho_{1}^{n} f\left(s_{1}^{n}\right)-\rho_{1}^{n} F\left(z_{2}^{n}, y^{n}\right)\right) \|\right) \\
\leq & \beta_{1}^{n} \zeta_{1}^{n}\left\|d_{1}^{n}-u^{n}\right\| \\
& +\beta_{1}^{n} \| R_{\rho_{1}^{A}, M}^{A, \eta_{1}}\left(A\left(u^{n}\right)+\rho_{1}^{n} f\left(u^{n}\right)-\rho_{1}^{n} F\left(z_{1}^{n}, y^{n}\right)\right) \\
& -R_{\rho_{1}^{n}, M}^{A, \eta_{1}}\left(A\left(s_{1}^{n}\right)+\rho_{1}^{n} f\left(s_{1}^{n}\right)-\rho_{1}^{n} F\left(z_{2}^{n}, y^{n}\right)\right) \| \\
\leq & \beta_{1}^{n} \zeta_{1}^{n}\left\|d_{1}^{n}-u^{n}\right\| \\
& +\beta_{1}^{n} \frac{\tau^{q-1}}{r_{1}-m_{1} \rho_{1}^{n}}\left[\rho_{1}^{n} \mu_{1} \gamma_{1}\left\|s_{1}^{n}-u^{n}\right\|+v_{1}\left\|y^{n}-y^{n}\right\|\right. \\
& \left.+\sqrt[q]{\omega_{1}^{q}+c_{q}\left(\rho_{1}^{n}\right)^{q} \kappa_{1}^{q}+q \sigma_{1} \omega_{1}^{q}-q \varphi_{1}}\left\|s_{1}^{n}-u^{n}\right\|\right]
\end{aligned}
$$


Li and Qu Fixed Point Theory and Applications 2012, 2012:195

Page 13 of 18

http://www.fixedpointtheoryandapplications.com/content/2012/1/195

$$
\begin{aligned}
\leq & \zeta_{1}^{n}\left\|x^{n+1}-x^{n}\right\|+\beta_{1}^{n} \zeta_{1}^{n}\left\|u^{n}-x^{n}\right\| \\
& +\beta_{1}^{n} \frac{\tau^{q-1}}{r_{1}-m_{1} \rho_{1}^{n}}\left[\rho_{1}^{n} \mu_{1} \gamma_{1}\left\|s_{1}^{n}-u^{n}\right\|+v_{1}\left\|y^{n}-y^{n}\right\|\right. \\
& \left.+\sqrt[q]{\omega_{1}^{q}+c_{q}\left(\rho_{1}^{n}\right)^{q} \kappa_{1}^{q}+q \sigma_{1} \omega_{1}^{q}-q \varphi_{1}}\left\|s_{1}^{n}-u^{n}\right\|\right] \\
& +\beta_{1}^{n} \frac{\tau^{q-1}}{r_{1}-m_{1} \rho_{1}^{n}}\left[\rho_{1}^{n} \mu_{1} \gamma_{1}+\sqrt[q]{\omega_{1}^{q}+c_{q}\left(\rho_{1}^{n}\right)^{q} \kappa_{1}^{q}+q \sigma_{1} \omega_{1}^{q}-q \varphi_{1}}\right]\left\|s_{1}^{n}-u^{n}\right\| \\
\leq & \zeta_{1}^{n}\left\|x^{n+1}-x^{*}\right\|+\zeta_{1}^{n}\left\|x^{n}-x^{*}\right\|+\beta_{1}^{n} \zeta_{1}^{n}\left\|u^{n}-x^{n}\right\| \\
& +\beta_{1}^{n} \frac{\tau^{q-1}}{r_{1}-m_{1} \rho_{1}^{n}}\left[\rho_{1}^{n} \mu_{1} \gamma_{1}+\sqrt[q]{\left.\omega_{1}^{q}+c_{q}\left(\rho_{1}^{n}\right)^{q} \kappa_{1}^{q}+q \sigma_{1} \omega_{1}^{q}-q \varphi_{1}\right]}\right. \\
& \times\left(\left\|s_{1}^{n}-x^{*}\right\|+\left\|x^{*}-x^{n}\right\|+\left\|x^{n}-u^{n}\right\|\right) \\
\leq & \zeta_{1}^{n}\left\|x^{n+1}-x^{*}\right\|+\left(\zeta_{1}^{n}+\beta_{1}^{n} \theta_{1}(n)\right)\left\|x^{n}-x^{*}\right\| \\
& +\beta_{1}^{n}\left(\zeta_{1}^{n}+\theta_{1}(n)\right)\left(\left\|u^{n}-x^{*}\right\|+\left\|x^{n}-x^{*}\right\|\right)+\beta_{1}^{n} \theta_{1}\left\|s_{1}^{n}-x^{*}\right\| \\
\leq & \zeta_{1}^{n}\left\|x^{n+1}-x^{*}\right\|+\left(\zeta_{1}^{n}+2 \beta_{1}^{n} \theta_{1}(n)+\beta_{1}^{n} \zeta_{1}^{n}\right)\left\|x^{n}-x^{*}\right\| \\
& +\beta_{1}^{n}\left(\zeta_{1}^{n}+\theta_{1}(n)\right)\left\|u^{n}-x^{*}\right\|+\beta_{1}^{n} \theta_{1}(n)\left\|s_{1}^{n}-x^{*}\right\| .
\end{aligned}
$$

It follows from (26)-(29) that

$$
\begin{aligned}
\left\|x^{n+1}-x^{*}\right\| \leq & \left\|x^{n+1}-t_{1}^{n+1}\right\|+\left\|t_{1}^{n+1}-x^{*}\right\| \\
\leq & \zeta_{1}^{n}\left\|x^{n+1}-x^{*}\right\|+\left[\zeta_{1}^{n}+2 \beta_{1}^{n} \theta_{1}(n)+\beta_{1}^{n} \zeta_{1}^{n}\right. \\
& +\beta_{1}^{n}\left(\zeta_{1}^{n}+\theta_{1}(n)\right) \frac{1}{1-\xi_{1}^{n}}\left(1-\alpha_{1}^{n}+\xi_{1}^{n}+\alpha_{1}^{n} \theta_{1}(n)\right) \\
& \left.+\left(1-\beta_{1}^{n}\right)\left(1-\alpha_{1}^{n}+\alpha_{1}^{n} \theta_{1}(n)\right)\right]\left\|x^{n}-x^{*}\right\| \\
& +\left[\beta_{1}^{n}\left(\zeta_{1}^{n}+\theta_{1}(n)\right) \frac{1}{1-\xi_{1}^{n}} \alpha_{1}^{n} \frac{\tau^{q-1}}{r_{1}-m_{1} \rho_{1}^{n}} v_{1}\right. \\
& \left.+\left(1-\beta_{1}^{n}\right) \beta_{1}^{n} \alpha_{1}^{n} \theta_{1}(n) \rho_{1}^{n} \nu_{1} \frac{\tau^{q-1}}{r_{1}-m_{1} \rho_{1}^{n}}\right]\left\|y^{n}-y^{*}\right\|,
\end{aligned}
$$

and

$$
\begin{aligned}
\left\|x^{n+1}-x^{*}\right\| \leq & \frac{1}{1-\zeta_{1}^{n}}\left[\zeta_{1}^{n}+2 \beta_{1}^{n} \theta_{1}(n)+\beta_{1}^{n} \zeta_{1}^{n}\right. \\
& +\beta_{1}^{n}\left(\zeta_{1}^{n}+\theta_{1}(n)\right) \frac{1}{1-\xi_{1}^{n}}\left(1-\alpha_{1}^{n}+\xi_{1}^{n}+\alpha_{1}^{n} \theta_{1}(n)\right) \\
& \left.+\left(1-\beta_{1}^{n}\right)\left(1-\alpha_{1}^{n}+\alpha_{1}^{n} \theta_{1}(n)\right)\right]\left\|x^{n}-x^{*}\right\| \\
& +\frac{1}{1-\zeta_{1}^{n}}\left[\beta_{1}^{n}\left(\zeta_{1}^{n}+\theta_{1}(n)\right) \frac{1}{1-\xi_{1}^{n}} \alpha_{1}^{n} \frac{\tau^{q-1}}{r_{1}-m_{1} \rho_{1}^{n}} v_{1}\right. \\
& \left.+\left(1-\beta_{1}^{n}\right) \beta_{1}^{n} \alpha_{1}^{n} \theta_{1}(n) \rho_{1}^{n} v_{1} \frac{\tau^{q-1}}{r_{1}-m_{1} \rho_{1}^{n}}\right]\left\|y^{n}-y^{*}\right\|,
\end{aligned}
$$


where

$$
\theta_{1}(n)=\frac{\tau^{q-1}}{r_{1}-m_{1} \rho_{1}^{n}}\left(\rho_{1}^{n} \mu_{1} \gamma_{1}+\sqrt[q]{\omega_{1}^{q}+c_{q}\left(\rho_{1}^{n}\right)^{q} \kappa_{1}^{q}+q \sigma_{1} \omega_{1}^{q}-q \varphi_{1}}\right) .
$$

For $n \geq 0$, we write

$$
\left\{\begin{aligned}
s_{2}^{n}= & \left(1-\alpha_{2}^{n}\right) y^{n}+\alpha_{2}^{n} R_{\rho_{2}^{n}, N}^{B, \eta_{2}}\left(B\left(y^{n}\right)+\rho_{2}^{n} g\left(y^{n}\right)-\rho_{2}^{n} G\left(w^{n}, x^{n}\right)\right) \\
& \left(w^{n} \in T\left(y^{n}\right)\right), \\
t_{2}^{n+1}= & \left(1-\beta_{2}^{n}\right) y^{n}+\beta_{2}^{n} R_{\rho_{2}^{n}, N}^{B, \eta_{2}}\left(B\left(s_{2}^{n}\right)+\rho_{2}^{n} g\left(s_{2}^{n}\right)-\rho_{2}^{n} G\left(w_{2}^{n}, x^{n}\right)\right) \\
& \left(w_{2}^{n} \in T\left(s_{2}^{n}\right)\right) .
\end{aligned}\right.
$$

By using the hypotheses of the mappings $B, g, G, T, N, \eta_{2}$ and $R_{\rho_{2}^{n}, N}^{B, \eta_{2}}$ in Theorem 5.1, and the same method as the one above, we can get

$$
\begin{aligned}
\left\|s_{2}^{n}-y^{*}\right\| \leq & \|\left(1-\alpha_{2}^{n}\right) y^{n}+\alpha_{2}^{n} R_{\rho_{2}^{n}, N}^{B, \eta_{2}}\left(B\left(y^{n}\right)+\rho_{2}^{n} g\left(y^{n}\right)-\rho_{2}^{n} G\left(w^{n}, x^{n}\right)\right. \\
& \left.-\left(1-\alpha_{2}^{n}\right) y^{*}-\alpha_{2}^{n} R_{\rho_{2}^{n}, N}^{B, \eta_{2}}\left(B\left(y^{*}\right)+\rho_{2} g\left(y^{*}\right)-\rho_{2} G\left(w^{*}, x^{*}\right)\right)\right) \| \\
\leq & \alpha_{2}^{n} \frac{\tau^{q-1}}{r_{2}-m_{2} \rho_{2}^{n}} \rho_{2}^{n} v_{2}\left\|x^{n}-x^{*}\right\|+\left[\left(1-\alpha_{2}^{n}\right)+\alpha_{2}^{n} \theta_{2}(n)\right]\left\|y^{n}-y^{*}\right\|, \\
\left\|v^{n}-y^{*}\right\| \leq & \|\left(1-\alpha_{2}^{n}\right) y^{n}+\alpha_{2}^{n} e_{2}^{n}-\left(1-\beta_{2}^{n}\right) y^{*} \\
& -\beta_{2}^{n} R_{\rho_{2}^{n}, N}^{B, \eta_{2}}\left(B\left(y^{*}\right)+\rho_{2}^{n} g\left(y^{*}\right)-\rho_{2}^{n} G\left(w^{*}, x^{*}\right)\right) \| \\
\leq & \left(1-\alpha_{2}^{n}\right)\left\|y^{n}-y^{*}\right\|+\alpha_{2}^{n}\left\|e_{2}^{n}-R_{\rho_{2}^{n}, N}^{B, \eta_{2}}\left(B\left(y^{n}\right)+\rho_{2}^{n} g\left(y^{n}\right)-\rho_{2}^{n} G\left(w^{n}, x^{n}\right)\right)\right\| \\
& +\alpha_{2}^{n} \| R_{\rho_{2}^{n}, N}^{B, \eta_{2}}\left(B\left(y^{n}\right)+\rho_{2}^{n} g\left(y^{n}\right)-\rho_{2}^{n} G\left(w^{n}, x^{n}\right)\right) \\
& -R_{\rho_{2}^{n}, N}^{B, \eta_{2}}\left(B\left(y^{*}\right)+\rho_{2}^{n} g\left(y^{*}\right)-\rho_{2}^{n} G\left(w^{*}, x^{*}\right)\right) \| \\
\leq & \left(1-\alpha_{2}^{n}\right)\left\|y^{n}-y^{*}\right\|+\alpha_{2}^{n} \xi_{2}^{n}\left\|e_{2}^{n}-y^{n}\right\| \\
& +\alpha_{2}^{n} \frac{\tau^{q-1}}{r_{2}-m_{2} \rho_{2}^{n}} \rho_{2}^{n} v_{2}\left\|x^{n}-x^{*}\right\|+\alpha_{2}^{n} \theta_{2}(n)\left\|y^{n}-y^{*}\right\| \\
\leq & \left(2-\alpha_{2}^{n}+\alpha_{2}^{n} \theta_{2}\right)\left\|y^{n}-y^{*}\right\|+\xi_{2}^{n}\left\|v^{n}-y^{*}\right\|+\alpha_{2}^{n} \frac{\tau^{q-1}}{r_{2}-m_{2} \rho_{2}^{n}} \rho_{2}^{n} \nu_{2}\left\|x^{n}-x^{*}\right\| ;
\end{aligned}
$$

that is,

$$
\begin{aligned}
\left\|s_{2}^{n}-y^{*}\right\| \leq & \alpha_{2}^{n} \frac{\tau^{q-1}}{r_{2}-m_{2} \rho_{2}^{n}} \rho_{2}^{n} v_{2}\left\|x^{n}-x^{*}\right\|+\left(1-\alpha_{2}^{n}+\alpha_{2}^{n} \theta_{2}(n)\right)\left\|y^{n}-y^{*}\right\|, \\
\left\|v^{n}-y^{*}\right\| \leq & \frac{1}{1-\xi_{2}^{n}}\left(2-\alpha_{2}^{n}+\alpha_{2}^{n} \theta_{2}(n)\right)\left\|y^{n}-y^{*}\right\| \\
& +\frac{1}{1-\xi_{2}^{n}} \alpha_{2}^{n} \rho_{2}^{n} v_{2} \frac{\tau^{q-1}}{r_{2}-m_{2} \rho_{2}^{n}}\left\|x^{n}-x^{*}\right\|,
\end{aligned}
$$

where $\alpha_{2}^{n}\left(e_{2}^{n}-y^{n}\right)=v^{n}-y^{n}$, and $\theta_{2}(n)=\frac{\tau^{q-1}}{r_{2}-m_{2} \rho_{2}^{n}}\left(\rho_{2}^{n} \mu_{2} \gamma_{2}+\sqrt[q]{\omega_{2}^{q}+c_{q}\left(\rho_{2}^{n}\right)^{q} \kappa_{2}^{q}+q \sigma_{2} \omega_{2}^{q}-q \varphi_{2}}\right)$. 
Moreover, we have

$$
\begin{aligned}
\left\|t_{2}^{n+1}-y^{*}\right\| \leq & \|\left(1-\beta_{2}^{n}\right) y^{n}+\beta_{2}^{n} d_{2}^{n}-\left(1-\beta_{2}^{n}\right) y^{*} \\
& -\beta_{2}^{n} R_{\rho_{2}^{n}, N}^{B, \eta_{2}}\left(B\left(y^{*}\right)+\rho_{2}^{n} g\left(y^{*}\right)-\rho_{2}^{n} G\left(w^{*}, x^{*}\right)\right) \| \\
\leq & \left(1-\beta_{2}^{n}\right)\left\|y^{n}-y^{*}\right\|+\beta_{2}^{n}\left\|d_{2}^{n}-R_{\rho_{2}^{n}, N}^{B, \eta_{2}}\left(B\left(v^{n}\right)+\rho_{2}^{n} g\left(v^{n}\right)-\rho_{2}^{n} G\left(w_{2}^{n}, x^{n}\right)\right)\right\| \\
& +\beta_{2}^{n} \| R_{\rho_{2}^{n}, N}^{B, \eta_{2}}\left(B\left(v^{n}\right)+\rho_{2}^{n} g\left(v^{n}\right)-\rho_{2}^{n} G\left(w_{2}^{n}, x^{n}\right)\right) \\
& -R_{\rho_{2}^{n}, N}^{B, \eta_{2}}\left(B\left(y^{*}\right)+\rho_{2}^{n} g\left(y^{*}\right)-\rho_{2}^{n} G\left(w^{*}, x^{*}\right)\right) \| \\
\leq & \left(1-\beta_{2}^{n}\right)\left\|y^{n}-y^{*}\right\|+\zeta_{2}^{n} \beta_{2}^{n}\left\|d_{2}^{n}-y^{n}\right\|+\beta_{2}^{n} \zeta_{2}^{n}\left\|y^{n}-s_{2}^{n}\right\| \\
& +\beta_{2}^{n} \frac{\tau^{q-1}}{r_{2}-m_{2} \rho_{2}^{n}} \rho_{2}^{n} v_{2}\left\|x^{n}-x^{*}\right\|+\beta_{2}^{n} \theta_{2}(n)\left\|v^{n}-y^{*}\right\| \\
\leq & \left(1-\beta_{2}^{n}+\zeta_{2}^{n}+\beta_{2}^{n} \zeta_{2}^{n}\right)\left\|y^{n}-y^{*}\right\|+\beta_{2}^{n} \frac{\tau^{q-1}}{r_{2}-m_{2} \rho_{2}^{n}} \rho_{2}^{n} v_{2}\left\|x^{n}-x^{* *}\right\| \\
& +\zeta_{2}^{n}\left\|y^{n+1}-y^{*}\right\|+\beta_{2}^{n} \zeta_{2}^{n}\left\|s_{2}^{n}-y^{*}\right\|+\beta_{2}^{n} \theta_{2}(n)\left\|v^{n}-y^{*}\right\|,
\end{aligned}
$$

for (19) $y^{n+1}-y^{n}=\beta_{2}^{n}\left(d_{2}^{n}-y^{n}\right)$.

It follows from (26) that

$$
\begin{aligned}
\left\|y^{n+1}-t_{2}^{n+1}\right\| \leq & \|\left(1-\beta_{2}^{n}\right) y^{n}+\beta_{2}^{n} d_{2}^{n}-\left(1-\beta_{2}^{n}\right) y^{n} \\
& -\beta_{2}^{n} R_{\rho_{2}^{n}, N}^{B, \eta_{2}}\left(B\left(s_{2}^{n}\right)+\rho_{2}^{n} g\left(s_{2}^{n}\right)-\rho_{2}^{n} G\left(w_{2}^{n}, x^{n}\right)\right) \| \\
\leq & \beta_{2}^{n}\left\|d_{2}^{n}-R_{\rho_{2}^{n}, N}^{B, \eta_{2}}\left(B\left(s_{2}^{n}\right)+\rho_{2}^{n} g\left(s_{2}^{n}\right)-\rho_{2}^{n} G\left(w_{2}^{n}, x^{n}\right)\right)\right\| \\
\leq & \beta_{2}^{n} \zeta_{2}^{n}\left\|d_{2}^{n}-v^{n}\right\|+\beta_{2}^{n} \frac{\tau^{q-1}}{r_{2}-m_{2} \rho_{2}^{n}} \rho_{2}^{n} v_{2}\left\|x^{n}-x^{n}\right\|+\beta_{2}^{n} \theta_{2}(n)\left\|s_{2}^{n}-v^{n}\right\| \\
\leq & \zeta_{2}^{n}\left(\left\|y^{n+1}-y^{*}\right\|+\left\|y^{n}-y^{*}\right\|\right)+\zeta_{2}^{n} \beta_{2}^{n}\left(\left\|y^{n}-y^{*}\right\|+\left\|v^{n}-y^{*}\right\|\right) \\
& +\beta_{2}^{n} \theta_{2}(n)\left(\left\|s_{2}^{n}-y^{*}\right\|+\left\|y^{*}-v^{n}\right\|\right) \\
\leq & \zeta_{2}^{n}\left\|y^{n+1}-y^{*}\right\|+\left(\zeta_{2}^{n}+\zeta_{2}^{n} \beta_{2}^{n}\right)\left\|y^{n}-y^{*}\right\| \\
& +\left(\zeta_{2}^{n} \beta_{2}^{n}+\beta_{2}^{n} \theta_{2}(n)\right)\left\|v^{n}-y^{*}\right\|+\beta_{2}^{n} \theta_{2}(n)\left\|s_{2}^{n}-y^{*}\right\| .
\end{aligned}
$$

Combining (30), (31), (32), (33) and (19), we have

$$
\begin{aligned}
\left\|y^{n+1}-y^{*}\right\| \leq & \frac{1}{1-2 \zeta_{2}^{n}}\left[\left(1-\beta_{2}^{n}+2 \beta_{2}^{n} \zeta_{2}^{n}+2 \zeta_{2}^{n}\right)\left\|y^{n}-y^{*}\right\|\right. \\
& +\beta_{2}^{n} \frac{\tau^{q-1}}{r_{2}-m_{2} \rho_{2}^{n}} \rho_{2}^{n} \nu_{2}\left\|x^{n}-x^{*}\right\|+\left(2 \beta_{2}^{n} \theta_{2}(n)+\zeta_{2}^{n} \beta_{2}^{n}\right)\left\|v^{n}-y^{*}\right\| \\
& \left.+\beta_{2}^{n}\left(\zeta_{2}^{n}+\theta_{2}(n)\right)\left\|s_{2}^{n}-y^{*}\right\|\right] \\
\leq & \frac{1}{1-2 \zeta_{2}^{n}}\left(1-\beta_{2}^{n}+2 \beta_{2}^{n} \zeta_{2}^{n}+2 \zeta_{2}^{n}\right. \\
& +\left(2 \beta_{2}^{n} \theta_{2}(n)+\zeta_{2}^{n} \beta_{2}^{n}\right) \frac{1}{1-\xi_{2}^{n}}\left(2-\alpha_{2}^{n}+\alpha_{2}^{n} \theta_{2}(n)\right)
\end{aligned}
$$




$$
\begin{aligned}
& \left.+\beta_{2}^{n}\left(\zeta_{2}^{n}+\theta_{2}(n)\right)\left[\left(1-\alpha_{2}^{n}\right)+\alpha_{2}^{n} \theta_{2}(n)\right]\right)\left\|y^{n}-y^{*}\right\| \\
& +\frac{1}{1-2 \zeta_{2}^{n}}\left(\beta_{2}^{n} \frac{\tau^{q-1}}{r_{2}-m_{2} \rho_{2}^{n}} \rho_{2}^{n} \nu_{2}+\beta_{2}^{n}\left(\zeta_{2}^{n}+\theta_{2}(n)\right) \alpha_{2}^{n} \frac{\tau^{q-1}}{r_{2}-m_{2} \rho_{2}^{n}} \rho_{2}^{n} \nu_{2}\right. \\
& \left.+\left(2 \beta_{2}^{n} \theta_{2}(n)+\zeta_{2}^{n} \beta_{2}^{n}\right) \frac{1}{1-\xi_{2}^{n}} \alpha_{2}^{n} \rho_{2}^{n} \nu_{2} \frac{\tau^{q-1}}{r_{2}-m_{2} \rho_{2}^{n}}\right)\left\|x^{n}-x^{*}\right\|
\end{aligned}
$$

By using (22) and (23), let

$$
\begin{aligned}
& a_{11}=\limsup _{n \rightarrow \infty} \frac{1}{1-\zeta_{1}^{n}}\left[\zeta_{1}^{n}+2 \beta_{1}^{n} \theta_{1}(n)+\beta_{1}^{n} \zeta_{1}^{n}\right. \\
& +\beta_{1}^{n}\left(\zeta_{1}^{n}+\theta_{1}(n)\right) \frac{1}{1-\xi_{1}^{n}}\left(1-\alpha_{1}^{n}+\xi_{1}^{n}+\alpha_{1}^{n} \theta_{1}(n)\right) \\
& \left.+\left(1-\beta_{1}^{n}\right)\left(1-\alpha_{1}^{n}+\alpha_{1}^{n} \theta_{1}(n)\right)\right] \\
& =1-\left(\alpha_{1}+\beta_{1}\right)+\left(\alpha_{1}+\beta_{1}\right) \theta_{1}(n)+\left(2-2 \alpha_{1}+\alpha_{1} \theta_{1}(n)\right) \beta_{1} \theta_{1}(n) \text {, } \\
& a_{12}=\limsup _{n \rightarrow \infty} \frac{1}{1-\zeta_{1}^{n}}\left[\beta_{1}^{n}\left(\zeta_{1}^{n}+\theta_{1}(n)\right) \frac{1}{1-\xi_{1}^{n}} \alpha_{1}^{n} \frac{\tau^{q-1}}{r_{1}-m_{1} \rho_{1}^{n}} v_{1}\right. \\
& \left.+\left(1-\beta_{1}^{n}\right) \beta_{1}^{n} \alpha_{1}^{n} \theta_{1}(n) \rho_{1}^{n} v_{1} \frac{\tau^{q-1}}{r_{1}-m_{1} \rho_{1}^{n}}\right] \\
& =\alpha_{1} \beta_{1} v_{1} \theta_{1} \frac{\tau^{q-1}}{r_{1}-m_{1} \rho_{1}}\left(1+\rho_{1}-\beta_{1} \rho_{1}\right) \text {, } \\
& a_{21}=\limsup _{n \rightarrow \infty} \frac{1}{1-2 \zeta_{2}^{n}}\left(\beta_{2}^{n} \frac{\tau^{q-1}}{r_{2}-m_{2} \rho_{2}^{n}} \rho_{2}^{n} v_{2}+\beta_{2}^{n}\left(\zeta_{2}^{n}+\theta_{2}(n)\right) \alpha_{2}^{n} \frac{\tau^{q-1}}{r_{2}-m_{2} \rho_{2}^{n}} \rho_{2}^{n} v_{2}\right. \\
& \left.+\left(2 \beta_{2}^{n} \theta_{2}(n)+\zeta_{2}^{n} \beta_{2}^{n}\right) \frac{1}{1-\xi_{2}^{n}} \alpha_{2}^{n} \rho_{2}^{n} \nu_{2} \frac{\tau^{q-1}}{r_{2}-m_{2} \rho_{2}^{n}}\right) \\
& =\beta_{2} \rho_{2} v_{2}\left(1+2 \alpha_{2} \theta_{2}\right) \frac{\tau^{q-1}}{r_{2}-m_{2} \rho_{2}} \text {, } \\
& a_{22}=\limsup _{n \rightarrow \infty} \frac{1}{1-2 \zeta_{2}^{n}}\left(1-\beta_{2}^{n}+2 \beta_{2}^{n} \zeta_{2}^{n}+2 \zeta_{2}^{n}\right. \\
& +\left(2 \beta_{2}^{n} \theta_{2}(n)+\zeta_{2}^{n} \beta_{2}^{n}\right) \frac{1}{1-\xi_{2}^{n}}\left(2-\alpha_{2}^{n}+\alpha_{2}^{n} \theta_{2}\right) \\
& \left.+\beta_{2}^{n}\left(\zeta_{2}^{n}+\theta_{2}(n)\right)\left[\left(1-\alpha_{2}^{n}\right)+\alpha_{2}^{n} \theta_{2}(n)\right]\right) \\
& =1-\beta_{2}+2 \beta_{2} \theta_{2}\left(2-\alpha_{2}+\alpha_{2} \theta_{2}\right)+\beta_{2} \theta_{2}\left(1-\alpha_{2}+\alpha_{2} \theta_{2}\right) \\
& =\left(1-\beta_{2}+\beta_{2} \theta_{2}\right)+3\left(1-\alpha_{2}+\alpha_{2} \theta_{2}\right) \beta_{2} \theta_{2}+\beta_{2} \theta_{2} \text {, }
\end{aligned}
$$

where

$$
\begin{aligned}
& \theta_{1}=\limsup _{n \rightarrow \infty} \theta_{1}(n)=\frac{\tau^{q-1}}{r_{1}-m_{1} \rho_{1}}\left(\rho_{1} \mu_{1} \gamma_{1}+l_{1}\right), \\
& \theta_{2}=\limsup _{n \rightarrow \infty} \theta_{2}(n)=\frac{\tau^{q-1}}{r_{2}-m_{2} \rho_{2}}\left(\rho_{2} \mu_{2} \gamma_{2}+l_{2}\right) .
\end{aligned}
$$


Let $\vec{A}=\left(\left\|x^{n+1}-x^{*}\right\|,\left\|y^{n+1}-y^{*}\right\|\right)^{T}$ and $\vec{B}=\left(\left\|x^{n}-x^{*}\right\|,\left\|y^{n}-y^{*}\right\|\right)^{T}$, then from (33), (34) and (35), we have $\vec{a} \leq \boldsymbol{\Psi} \vec{b}$, where

$$
\boldsymbol{\Psi}=\left(\begin{array}{ll}
a_{11} & a_{12} \\
a_{21} & a_{22}
\end{array}\right)
$$

which is called the matrix for a nonlinear set-valued inclusions system involving $(A, \eta)$ accretive mappings. By using [16], we have

$$
\|\vec{A}\| \leq\|\Psi\|\|\vec{B}\|
$$

Let

$$
\|\boldsymbol{\Psi}\|=\max \left\{a_{11}, a_{12}, a_{21}, a_{22}\right\}
$$

It follows from (21)-(23), Theorem 3.1 and [15] that $0<\|\boldsymbol{\Psi}\|<1$ and there exist $x^{*}, y^{*} \in X$ and $z^{*} \in S\left(x^{*}\right), w^{*} \in T\left(y^{*}\right)[17]$ such that

$$
\left\{\begin{array}{l}
Q_{1}\left(x^{*}\right)=x^{*} \\
Q_{2}\left(y^{*}\right)=y^{*}
\end{array}\right.
$$

and the sequence $\left\{x^{n}, y^{n}\right\}_{n=0}^{\infty}$ generated by Ishikawa-hybrid proximal Algorithm 4.1 converges linearly to a solution $\left(x^{*}, y^{*}\right)$ of the problem (3) as the convergence rate

$$
\begin{aligned}
\|\boldsymbol{\Psi}\|= & \max \left\{1-\left(\alpha_{1}+\beta_{1}\right)+\left(\alpha_{1}+\beta_{1}\right) \theta_{1}+\left(2-2 \alpha_{1}+\alpha_{1} \theta_{1}\right) \beta_{1} \theta_{1},\right. \\
& \alpha_{1} \beta_{1} v_{1} \theta_{1} \frac{\tau^{q-1}}{r_{1}-m_{1} \rho_{1}}\left(1+\rho_{1}-\beta_{1} \rho_{1}\right), \\
& 1-\beta_{2}+2 \beta_{2} \theta_{2}\left(2-\alpha_{2}+\alpha_{2} \theta_{2}\right)+\beta_{2} \theta_{2}\left(1-\alpha_{2}+\alpha_{2} \theta_{2}\right), \\
& \left.\left(1-\beta_{2}+\beta_{2} \theta_{2}\right)+3\left(1-\alpha_{2}+\alpha_{2} \theta_{2}\right) \beta_{2} \theta_{2}+\beta_{2} \theta_{2}\right\},
\end{aligned}
$$

where $c_{q}>0$ is the same as in Lemma 2.7, $\rho_{i} \in\left(0, \frac{r_{i}}{m_{i}}\right)(i=1,2)$. This completes the proof.

Remark 5.2 For a suitable choice of the mappings $A, B, \eta_{i}, F, G, M, N, S, T, f, g$ and $X$, we can obtain several known results in $[7,9,11,12]$ as special cases of Theorem 5.1 . 


\section{References}

1. Hassouni, A, Moudafi, A: A perturbed algorithms for variational inequalities. J. Math. Anal. Appl. 185, 706-712 (2001)

2. Kassay, G, Kolumbán, J: System of multi-valued variational inequalities. Publ. Math. (Debr.) 54, 267-279 (1999)

3. Chen, XF, Deng, CX, Tan, MY: New approximation algorithm for a system of generalized nonlinear variational inequalities. J. Sichuan Univ. Nat. Sci. Ed. 38(6), 813-817 (2001)

4. Yan, WY, Fang, YP, Huang, NJ: A new system of set-valued variational inclusions with $\mathrm{H}$-monotone operators. Math. Inequal. Appl. 8(3), 537-546 (2005)

5. Fang, YP, Huang, NJ, Thompson, HB: A new system of variational inclusions with $(H, \eta)$-monotone operators in Hilbert spaces. Comput. Math. Appl. 49, 365-374 (2005)

6. Jin, MM: Approximation algorithm for a class of generalized nonlinear set-valued variational inclusions systems. J. Sichuan Norm. Univ. Nat. Sci. 29, 1-4 (2006)

7. Verma, RU: A hybrid proximal point algorithm based on the $(A, \eta)$-maximal monotonicity framework. Appl. Math. Lett. 21, 142-147 (2008)

8. $L i, H G, X u, A J$, Jin, MM: A hybrid proximal point three-step algorithm for nonlinear set-valued quasi-variational inclusions system involving $(A, \eta)$-accretive mappings. Fixed Point Theory Appl. 2010, Article ID 635382 (2010). doi:10.1155/2010/635382

9. Kang, SM, Cho, SY, Liu, Z: Convergence of iterative sequences for generalized equilibrium problems involving inverse-strongly monotone mappings. J. Inequal. Appl. 2010, Article ID 827082 (2010)

10. Lan, $H Y$, Cho, YJ, Verma, RU: On nonlinear relaxed cocoercive inclusions involving $(A, \eta)$-accretive mappings in Banach spaces. Comput. Math. Appl. 51, 1529-1538 (2006)

11. Eckstein, J, Bertsekas, DP: On the Douglas-Rachford splitting method and the proximal point algorithm for maximal monotone operators. Math. Program. 55, 293-318 (1992)

12. $L i, H G, X u, A J$, Jin, MM: A Ishikawa-hybrid proximal point algorithm for nonlinear set-valued inclusions problem based on $(A, \eta)$-accretive framework. Fixed Point Theory Appl. 2010, Article ID 501293 (2010). doi:10.1155/2010/501293

13. Zou, YZ, Huang, NJ: A new system of variational inclusions involving $H(\cdot, \cdot)$-accretive operator in Banach spaces. Appl. Math. Comput. 212, 135-144 (2009)

14. Xu, HK: Inequalities in Banach spaces with applications. Nonlinear Anal. 16(12), 1127-1138 (1991)

15. Nadler, SB: Multi-valued contraction mappings. Pac. J. Math. 30, 475-488 (1969)

16. Horn, RA, Johnson, CR: Matrix Analysis. Cambridge University Press, Cambridge (1986)

17. Li, HG: Iterative algorithm for a new class of generalized nonlinear fuzzy set-valued variational inclusions involving $(H, \eta)$-monotone mappings. Adv. Nonlinear Var. Inequal. 10(1), 41-50 (2007)

18. Peng, JW, Zhu, DL: Three-step iterative algorithm for a system of set-valued variational inclusions with $(H, \eta)$-monotone operators. Nonlinear Anal. 68, 139-153 (2008)

19. Cohen, G, Chaplais, F: Nested monotony for variational inequalities over a product of spaces and convergence of iterative algorithms. J. Optim. Theory Appl. 59, 360-390 (1988)

20. Ansari, QH, Yao, JC: A fixed point theorem and its applications to a system of variational inequalities. Bull. Aust. Math. Soc. 59, 433-442 (1999)

21. Allevi, E, Gnudi, A, Konnov, IV: Generalized vector variational inequalities over product sets. Nonlinear Anal. 47, 573-582 (2001)

22. Kassay, G, Kolumbán, J: System of multi-valued variational inequalities. Publ. Math. (Debr.) 54, $267-279$ (1999)

23. Kim, JK, Kim, DS: A new system of generalized nonlinear mixed variational inequalities in Hilbert spaces. J. Convex Anal. 11(1), 235-243 (2004)

24. Li, HG: Approximation solutions for generalized multi-valued variational-like inclusions with $(G, \eta)$-monotone mappings. J. Jishou Univ. Nat. Sci. Ed. 30(4), 7-12 (2009)

25. Cho, SY, Kang, SM: Approximation of fixed points of pseudocontraction semigroups based on a viscosity iterative process. Appl. Math. Lett. 24, 224-228 (2011)

26. Ye, J, Huang, J: Strong convergence theorems for fixed point problems and generalized equilibrium problems of three relatively quasi-nonexpansive mappings in Banach spaces. J. Math. Comput. Sci. 1, 1-18 (2011)

27. Yang, S, Li, W: Iterative solutions of a system of equilibrium problems in Hilbert spaces. Adv. Fixed Point Theory 1 , 15-26 (2011)

28. Chang, SS, Chan, CK, Lee, HWJ, Yang, L: A system of mixed equilibrium problems, fixed point problems of strictly pseudo-contractive mappings and nonexpansive semi-groups. Appl. Math. Comput. 216, 51-60 (2010)

doi:10.1186/1687-1812-2012-195

Cite this article as: Li and Qiu: Ishikawa-hybrid proximal point algorithm for NSVI system. Fixed Point Theory and Applications 2012 2012:195. 